\title{
Scattering phaseshift formulas for mesons and baryons in elon- gated boxes
}

\author{
Frank X. Lee ${ }^{1, \star}$ and Andrei Alexandru ${ }^{1,2}$ \\ ${ }^{1}$ Physics Department, The George Washington University, Washington, DC 20052, USA \\ ${ }^{2}$ Albert Einstein Center for Fundamental Physics, Institute for Theoretical Physics, University of Bern, Sidler- \\ strasse 5, CH-3012 Bern, Switzerland
}

\begin{abstract}
We derive Lüscher phaseshift formulas for two-particle states in boxes elongated in one of the dimensions. Such boxes offer a cost-effective way of varying the relative momentum of the particles. Boosted states in the elongated direction, which allow wider access to energies, are also considered. The formulas for the various scenarios (moving and zero-momentum states in cubic and elongated boxes) are compared and relations between them are clarified. The results are applicable to a wide set of meson-meson and meson-baryon elastic scattering processes, with the two-particle system having equal or unequal masses.
\end{abstract}

\section{Introduction}

Hadron-hadron scattering is an indispensable tool in understanding the nature of the strong nuclear force, both experimentally and theoretically. The theoretical groundwork was laid out by Lüscher [1] who showed how to relate elastic scattering phaseshifts with the energies of the two-body states in a finite box. Various extensions to the method have since been made to enhance its applications, including moving frames [2], moving frame involving unequal masses and baryons [3-5], asymmetric boxes [6], and more recently inelastic scattering [7, 8]. The use of asymmetric boxes has proven to be efficient in recent studies of the $\rho$ meson resonance in $\pi \pi$ scattering $[9,10]$. Instead of varying the size of the entire box, only one side is elongated, requiring much less computing resources. Our main goal in this work is to derive the phaseshift formulas needed to study meson-baryon elastic scattering in elongated boxes, with an eye towards a lattice QCD simulation of the $\Delta$ resonance in $\pi N$ scattering.

\section{Angular momentum resolution}

We consider a box elongated in the $z$-direction as illustrated in Fig. 1. The infinite volume symmetry group for spatial rotations is $S O(3)$ which has an infinite number of elements and irreducible representations (irreps) labeled by angular momentum $J$. For spherically symmetric interactions the eigenstates of the Hamiltonian in the infinite volume form multiplets that furnish bases for the irreps of $S U(2)$, the double cover of the rotations group. These multiplets are labeled by the angular momentum $J=0, \frac{1}{2}, 1, \frac{3}{2}, 2, \ldots$. For elongated boxes, these multiplets split into smaller sets that mix

${ }^{\star}$ Speaker, e-mail: fxlee@gwu.edu 


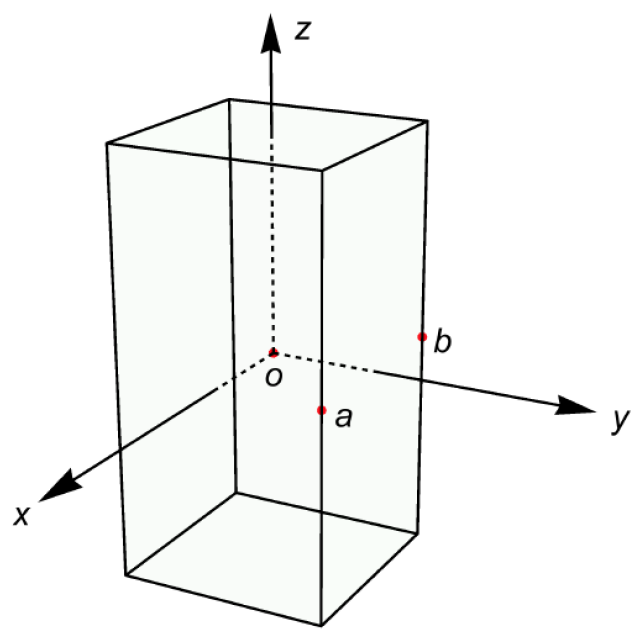

(a) The 8 symmetry operations that form the dihedral $D_{4}$ group in the elongated box whose dimensions are $L \times L \times \eta L$ where $\eta$ is the elongation factor in the $z$-direction.

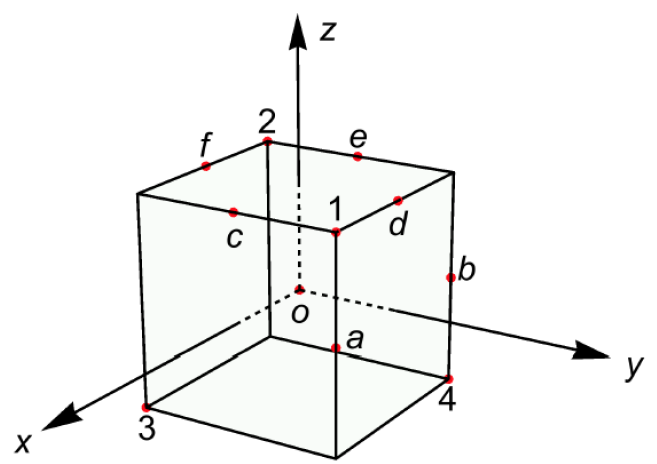

(b) The 24 symmetry operations in the cubic box that form the octahedral group $O$.

Figure 1. Elongated vs. cubic proper group symmetries. The full symmetry must include space inversion (parity), and their double-cover groups for half-integer angular momentum.

under the action of rotations that leave the box invariant, forming the bases for one of the 7 irreps of the ${ }^{2} D_{4}$ group. Then the question is: for a given $J$, what irreps are coupled to it? To answer this we have to decompose the irrep $J$ of the full rotation group $S U(2)$, into a direct sum of the irreps of the ${ }^{2} D_{4}$ group, $J=\bigoplus_{i} n\left(\Gamma_{i}, J\right) \Gamma_{i}$, where the coefficient is called the multiplicity, which tells how many times irrep $\Gamma_{i}$ appears in the given $J$. This can be calculated using

$$
n\left(\Gamma_{i}, J\right)=\frac{1}{g} \sum_{k} n_{k} \chi\left(k, \Gamma_{i}\right) \chi\left(\omega_{k}, J\right) .
$$

The index $k$ runs through all 7 classes of ${ }^{2} D_{4}$ and $g=16$ is the total number of elements in the group. $n_{k}$ is the number of elements in the $k$-th class, and $\chi\left(k, \Gamma_{i}\right)$ are the characters. The $\chi\left(\omega_{k}, J\right)$ stands for the character of full rotation group for angular momentum $J$ and rotation angle $\omega_{k}$ in class $k$. This can be computed as follows. Any rotation $k$ is characterized by a rotation axis and the rotation angle $\omega_{k}$. Since the character (trace) of the matrix is invariant under similarity transformations the result will be equal to an equivalent rotation around the $z$-axis (the similarity matrix in this case is simply a rotation that takes the rotation axis into the $z$-axis). The character is then the trace of this diagonal matrix

$$
\chi\left(\omega_{k}, J\right)=\sum_{m=-J}^{J} e^{-i m \omega_{k}}=\frac{\sin \left[(J+1 / 2) \omega_{k}\right]}{\sin \left(\omega_{k} / 2\right)} .
$$

The results of the decomposition from applying Eq. 1 are given in Table 1. For comparison, Table 2 shows the angular momentum resolution in the cubic box. 
Table 1. Decomposition of angular momentum in the elongated box according to the irreps of the ${ }^{2} D_{4 h}$ group. Both the original reduction (left) and its inverse (right) are shown. Parity is indicated by the plus (even) or minus (odd) sign.

\begin{tabular}{cc|ll}
\hline$J$ & ${ }^{2} D_{4 h}$ & ${ }^{2} D_{4 h}$ & $J$ \\
\hline 0 & $A_{1}^{+}$ & $A_{1}^{+}$ & $0,2,4(2), \cdots$ \\
1 & $A_{2}^{-} \oplus E^{-}$ & $A_{1}^{-}$ & $5,7,9(2), \cdots$ \\
2 & $A_{1}^{+} \oplus B_{1}^{+} \oplus B_{2}^{+} \oplus E^{+}$ & $A_{2}^{-}$ & $1,3,5(2), \cdots$ \\
3 & $A_{2}^{-} \oplus B_{1}^{-} \oplus B_{2}^{-} \oplus 2 E^{-}$ & $A_{2}^{+}$ & $4,6,8(2), \cdots$ \\
4 & $2 A_{1}^{+} \oplus A_{2}^{+} \oplus B_{1}^{+} \oplus B_{2}^{+} \oplus 2 E^{+}$ & $B_{1}^{+}$ & $2,4,6(2), \cdots$ \\
5 & $A_{1}^{-} \oplus 2 A_{2}^{-} \oplus B_{1}^{-} \oplus B_{2}^{-} \oplus 3 E^{-}$ & $B_{1}^{-}$ & $3,5,7(2), \cdots$ \\
6 & $2 A_{1}^{+} \oplus A_{2}^{+} \oplus 2 B_{1}^{+} \oplus 2 B_{2}^{+} \oplus 3 E^{+}$ & $B_{2}^{+}$ & $2,4,6(2), \cdots$ \\
& & $B_{2}^{-}$ & $3,5,7(2), \cdots$ \\
$\cdots$ & $\ldots$ & $E^{-}$ & $1,3(2), 5(3), \cdots$ \\
& & $E^{+}$ & $2,4(2), 6(3), \cdots$ \\
& & & \\
$1 / 2$ & $G_{1}^{ \pm}$ & $G_{1}^{ \pm}$ & $1 / 2,3 / 2,5 / 2, \cdots$ \\
$3 / 2$ & $G_{1}^{ \pm} \oplus G_{2}^{ \pm}$ & $G_{2}^{ \pm}$ & $3 / 2,5 / 2(2), 7 / 2(2), \cdots$ \\
$5 / 2$ & $G_{1}^{ \pm} \oplus 2 G_{2}^{ \pm}$ & & \\
$7 / 2$ & $2 G_{1}^{ \pm} \oplus 2 G_{2}^{ \pm}$ & & \\
$\cdots$ & $\cdots$ & & \\
\hline
\end{tabular}

Table 2. Decomposition of angular momentum in the cubic box according to the irreps of the ${ }^{2} O_{h}$ group. Both the original decomposition (left) and its inverse (right) are shown. The number in parentheses indicates the multiplicity of that $J$ in that irrep.

\begin{tabular}{cc|ll}
\hline$J$ & ${ }^{2} O_{h}$ & ${ }^{2} O_{h}$ & $\mathrm{~J}$ \\
\hline 0 & $A_{1}^{+}$ & $A_{1}^{+}$ & $0,4,6, \cdots$ \\
1 & $T_{1}^{-}$ & $A_{1}^{-}$ & $9,13,15, \cdots$ \\
2 & $T_{2}^{+} \oplus E^{+}$ & $T_{1}^{-}$ & $1,3,5(2), \cdots$ \\
3 & $A_{2}^{-} \oplus T_{1}^{-} \oplus T_{2}^{-}$ & $T_{1}^{+}$ & $4,6,8(2), \cdots$ \\
4 & $A_{1}^{+} \oplus E^{+} \oplus T_{1}^{+} \oplus T_{2}^{+}$ & $T_{2}^{+}$ & $2,4,6(2), \cdots$ \\
5 & $E^{-} \oplus 2 T_{1}^{-} \oplus T_{2}^{-}$ & $T_{2}^{-}$ & $3,5,7(2), \cdots$ \\
6 & $A_{1}^{+} \oplus A_{2}^{+} \oplus E^{+} \oplus T_{1}^{+} \oplus 2 T_{2}^{+}$ & $E^{+}$ & $2,4,6, \cdots$ \\
& & $E^{-}$ & $5,7,9, \cdots$ \\
$\cdots$ & $\cdots$ & $A_{2}^{-}$ & $3,7,9, \cdots$ \\
& & $A_{2}^{+}$ & $6,10,12, \cdots$ \\
& & & \\
$1 / 2$ & $G_{1}^{ \pm}$ & $G_{1}^{ \pm}$ & $1 / 2,7 / 2,9 / 2, \cdots$ \\
$3 / 2$ & $H^{ \pm}$ & $H^{ \pm}$ & $3 / 2,5 / 2,7 / 2, \cdots$ \\
$5 / 2$ & $G_{2}^{ \pm} \oplus H^{ \pm}$ & $G_{2}^{ \pm}$ & $5 / 2,7 / 2,11 / 2, \cdots$ \\
$7 / 2$ & $G_{1}^{ \pm} \oplus G_{2}^{ \pm} \oplus H^{ \pm}$ & & \\
$\cdots$ & $\cdots$ & & \\
\hline
\end{tabular}




\subsection{Phaseshift formulas in the elongated box}

Our starting point is

$$
\operatorname{det}\left[\mathcal{M}_{J l M, J^{\prime} l^{\prime} M^{\prime}}-\delta_{J J^{\prime}} \delta_{l l^{\prime}} \delta_{M M^{\prime}} \cot \delta_{J l}\right]=0,
$$

expressed for a given total angular momentum $J$ and partial-wave $l$. The matrix $\mathcal{M}$ is adapted from the original one by Lüscher for integer angular momentum, cubic boxes, and equal masses to the current case of half-integer angular momentum, elongated boxes (limited to $z$-direction), and unequal masses. The projection to half-integer angular momentum is achieved by a straightforward change of basis by coupling to spin- $1 / 2$,

$$
\mathcal{M}_{J l M, J^{\prime} l^{\prime} M^{\prime}}=\sum_{m m^{\prime} m_{s} m_{s}^{\prime}}\left\langle l m, \frac{1}{2} m_{s} \mid J M\right\rangle\left\langle l^{\prime} m^{\prime}, \frac{1}{2} m_{s}^{\prime} \mid J^{\prime} M^{\prime}\right\rangle \mathcal{M}_{l m, l^{\prime} m^{\prime}},
$$

using Clebsch-Gordan coefficients. The modified matrix for $z$-elongated box (of elongation $\eta$ ) is

$$
\mathcal{M}_{l m, l^{\prime} m^{\prime}}(q, \eta)=\sum_{j=\left[l-l^{\prime} \mid\right.}^{l+l^{\prime}} \sum_{s=-j}^{j} \frac{(-1)^{l} l^{l+l^{\prime}}}{\pi^{3 / 2} \eta q^{j+1}} Z_{j s}\left(1, q^{2}, \eta\right) \times\left\langle l 0 j 0 \mid l^{\prime} 0\right\rangle\left\langle l m j s \mid l^{\prime} m^{\prime}\right\rangle \sqrt{\frac{(2 l+1)(2 j+1)}{\left(2 l^{\prime}+1\right)}} .
$$

It is customary to introduce the short-hand function for the zeta function,

$$
\mathcal{W}_{l m}\left(1, q^{2} ; \eta\right)=\frac{\mathcal{Z}_{l m}\left(1, q^{2} ; \eta\right)}{\pi^{3 / 2} \eta q^{l+1}}, \text { where } \mathcal{Z}_{l m}\left(s, q^{2} ; \eta\right)=\sum_{\boldsymbol{n} \in Z^{3}} \frac{\mathcal{Y}_{l m}(\widetilde{\boldsymbol{n}})}{\left(\widetilde{\boldsymbol{n}}^{2}-q^{2}\right)^{s}},
$$

so the simplest phaseshift formula reads $\cot \delta=\mathcal{W}_{00}$. The $\mathcal{M}$ matrix is a linear combination of $\mathcal{W}$ functions with purely numerical coefficients. The dimensionless momentum $\boldsymbol{q}$ is defined in terms of the minimal momentum in a periodic box of size $L, \boldsymbol{k}=(2 \pi / L) \boldsymbol{q}$. The $\mathcal{Y}_{l m}(\boldsymbol{r}) \equiv r^{l} Y_{l m}(\theta, \phi)$ are homogenous harmonic polynomials and the modified index $\widetilde{\boldsymbol{n}}$ is related to the cubic index $\boldsymbol{n}=$ $\left(n_{x}, n_{y}, n_{z}\right)$ by $\widetilde{\boldsymbol{n}}=\left(n_{x}, n_{y}, n_{z} / \eta\right)$. Details on how to numerically evaluate the function with elongation can be found in Refs. [6, 9].

The spin-projected matrix $\mathcal{M}_{J l M, J^{\prime} l^{\prime} M^{\prime}}$ is still expressed in terms of angular momentum labels $J l M$. Our goal is to reduce the matrix to the irreps of the ${ }^{2} D_{4 h}$ group in elongated boxes. Operationally, it is equivalent to the reduction of the matrix into its block diagonal form with each block having the dimension of an irrep. This is achieved by another change of basis, using the basis vectors adapted to the symmetry groups. In the new basis, $\mathcal{M}$ is block-diagonalized by irreps

$$
\left\langle\Gamma \alpha J l n|\mathcal{M}| \Gamma^{\prime} \alpha^{\prime} J^{\prime} l^{\prime} n^{\prime}\right\rangle=\sum_{M M^{\prime}}\left(C_{J l M}^{\Gamma \alpha n}\right)^{*} C_{J^{\prime} l^{\prime} M^{\prime}}^{\Gamma^{\prime} \alpha^{\prime}{ }^{\prime}} \mathcal{M}_{J l M, J^{\prime} l^{\prime} M^{\prime}}=\delta_{\Gamma \Gamma^{\prime}} \delta_{\alpha \alpha^{\prime}} \mathcal{M}_{J l n, J^{\prime} l^{\prime} n^{\prime}}^{\Gamma}
$$

where Schur's lemma in linear algebra was used in the second step. For multi-dimensional irreps, the matrix is diagonal in $\alpha$ and the quantization condition does not depend on it. The final form for the phaseshift reduction is

$$
\prod_{\Gamma} \operatorname{det}\left[\mathcal{M}_{J l n, J^{\prime} l^{\prime} n^{\prime}}^{\Gamma}-\delta_{J J^{\prime}} \delta_{l l^{\prime}} \delta_{n n^{\prime}} \cot \delta_{J l}\right]=0 .
$$

If there is no multiplicity, the labels $n$ and $n^{\prime}$ can be dropped. For half-integral angular momentum up to $J=7 / 2$ our results for the two irreps $G_{1}$ and $G_{2}$ are new and are given in Table 3 . There is two-fold multiplicity for $J=7 / 2$ so the $n$ and $n^{\prime}$ labels are kept explicit.

The final step is to determine the phaseshift using the matrix elements. We will not show results for all the irreps, but only illustrate the treatment in a few examples. For half-integral angular momentum, 
Table 3. Non-zero reduced matrix elements in the elongated box $\left({ }^{2} D_{4 h}\right.$ symmetry group) for half-integral angular momentum up to $J=7 / 2$. The even/odd parity sectors are indicated by the upper/lower signs. The matrix is symmetric in $J n$ and $J^{\prime} n^{\prime}$ in each irrep-parity sector.

\begin{tabular}{|c|c|c|c|c|c|}
\hline$\Gamma$ & $J$ & $n$ & $J^{\prime}$ & $n^{\prime}$ & $\mathcal{M}_{J l n, J^{\prime} l^{\prime} n^{\prime}}^{\Gamma}$ \\
\hline \multirow[t]{15}{*}{$G_{1}^{ \pm}$} & $\frac{1}{2}$ & 1 & $\frac{1}{2}$ & 1 & $\mathcal{W}_{00}$ \\
\hline & $\frac{1}{2}$ & 1 & $\frac{3}{2}$ & 1 & $\pm \sqrt{\frac{2}{5}} \boldsymbol{W}_{20}$ \\
\hline & $\frac{1}{2}$ & 1 & $\frac{5}{2}$ & 1 & $-\sqrt{\frac{3}{5}} \boldsymbol{W}_{20}$ \\
\hline & $\frac{1}{2}$ & 1 & $\frac{7}{2}$ & 1 & $-\frac{2}{3} \mathcal{W}_{40}$ \\
\hline & $\frac{1}{2}$ & 1 & $\frac{7}{2}$ & 2 & $\mp \frac{2}{3} \sqrt{2} W_{44}$ \\
\hline & $\frac{3}{2}$ & 1 & $\frac{3}{2}$ & 1 & $\mathcal{W}_{00}+\frac{1}{\sqrt{5}} \boldsymbol{W}_{20}$ \\
\hline & $\frac{3}{2}$ & 1 & $\frac{5}{2}$ & 1 & $\mp \frac{1}{7} \sqrt{\frac{6}{5}} \boldsymbol{W}_{20} \mp \frac{2 \sqrt{6}}{7} \boldsymbol{W}_{40}$ \\
\hline & $\frac{3}{2}$ & 1 & $\frac{7}{2}$ & 1 & $-\frac{9}{7} \sqrt{\frac{2}{5}} \mathcal{W}_{20}-\frac{5 \sqrt{2}}{21} \boldsymbol{W}_{40}$ \\
\hline & $\frac{3}{2}$ & 1 & $\frac{7}{2}$ & 2 & $\frac{2}{3} \mathcal{W}_{44}$ \\
\hline & $\frac{5}{2}$ & 1 & $\frac{5}{2}$ & 1 & $\boldsymbol{W}_{00}+\frac{8}{7 \sqrt{5}} \boldsymbol{W}_{20}+\frac{2}{7} \boldsymbol{W}_{40}$ \\
\hline & $\frac{5}{2}$ & 1 & $\frac{7}{2}$ & 1 & $\pm \frac{2}{7 \sqrt{15}} \mathcal{W}_{20} \pm \frac{10 \sqrt{3}}{77} \mathcal{W}_{40} \pm \frac{50}{11 \sqrt{39}} \mathcal{W}_{60}$ \\
\hline & $\frac{5}{2}$ & 1 & $\frac{7}{2}$ & 2 & $\mp \frac{2 \sqrt{6}}{11} \mathcal{W}_{44} \pm \frac{10}{11} \sqrt{\frac{10}{39}} \mathcal{W}_{64}$ \\
\hline & $\frac{7}{2}$ & 1 & $\frac{7}{2}$ & 1 & $\mathcal{W}_{00}+\frac{5}{21} \sqrt{5} \mathcal{W}_{20}+\frac{27}{77} \mathcal{W}_{40}+\frac{25 \mathcal{W}_{60}}{33 \sqrt{13}}$ \\
\hline & $\frac{7}{2}$ & 1 & $\frac{7}{2}$ & 2 & $\frac{3 \sqrt{2}}{11} \mathcal{W}_{44}-\frac{5}{11} \sqrt{\frac{10}{13}} \mathcal{W}_{64}$ \\
\hline & $\frac{7}{2}$ & 2 & $\frac{7}{2}$ & 2 & $\mathcal{W}_{00}-\frac{\sqrt{5}}{3} \mathcal{W}_{20}+\frac{3}{11} \mathcal{W}_{40}-\frac{5}{33 \sqrt{13}} \mathcal{W}_{60}$ \\
\hline \multirow[t]{15}{*}{$G_{2}^{ \pm}$} & $\frac{3}{2}$ & 1 & $\frac{3}{2}$ & 1 & $\mathcal{W}_{00}-\frac{1}{\sqrt{5}} \boldsymbol{W}_{20}$ \\
\hline & $\frac{3}{2}$ & 1 & $\frac{5}{2}$ & 1 & $\pm \frac{6}{7 \sqrt{5}} \boldsymbol{W}_{20} \mp \frac{2}{7} \boldsymbol{W}_{40}$ \\
\hline & $\frac{3}{2}$ & 1 & $\frac{5}{2}$ & 2 & $\mp 2 \sqrt{\frac{2}{7}} \mathcal{W}_{44}$ \\
\hline & $\frac{3}{2}$ & 1 & $\frac{7}{2}$ & 1 & $-\frac{3 \sqrt{2}}{7} \mathcal{W}_{20}+\frac{\sqrt{10}}{7} \mathcal{W}_{40}$ \\
\hline & $\frac{3}{2}$ & 1 & $\frac{7}{2}$ & 2 & $\frac{2}{\sqrt{21}} W_{44}$ \\
\hline & $\frac{5}{2}$ & 1 & $\frac{5}{2}$ & 1 & $\boldsymbol{W}_{00}+\frac{2}{7 \sqrt{5}} \boldsymbol{W}_{20}-\frac{3}{7} \boldsymbol{W}_{40}$ \\
\hline & $\frac{5}{2}$ & 1 & $\frac{5}{2}$ & 2 & $\sqrt{\frac{2}{7}} \mathcal{W}_{44}$ \\
\hline & $\frac{5}{2}$ & 2 & $\frac{5}{2}$ & 2 & $\mathcal{W}_{00}-\frac{2 \sqrt{5}}{7} \boldsymbol{W}_{20}+\frac{1}{7} \boldsymbol{W}_{40}$ \\
\hline & $\frac{5}{2}$ & 1 & $\frac{7}{2}$ & 1 & $\pm \frac{\sqrt{2}}{7} \mathcal{W}_{20} \pm \frac{8 \sqrt{10}}{77} \mathcal{W}_{40} \mp \frac{5}{11} \sqrt{\frac{10}{13}} \mathcal{W}_{60}$ \\
\hline & $\frac{5}{2}$ & 1 & $\frac{7}{2}$ & 2 & $\mp \frac{8}{11} \sqrt{\frac{3}{7}} \mathcal{W}_{44} \mp \frac{10}{11} \sqrt{\frac{35}{39}} \mathcal{W}_{64}$ \\
\hline & $\frac{5}{2}$ & 2 & $\frac{7}{2}$ & 1 & $\pm \frac{4}{11} \sqrt{\frac{5}{7}} \mathcal{W}_{44} \mp \frac{10}{11} \sqrt{\frac{7}{13}} \boldsymbol{W}_{64}$ \\
\hline & $\frac{5}{2}$ & 2 & $\frac{7}{2}$ & 2 & $\mp \frac{1}{7} \sqrt{\frac{10}{3}} \mathcal{W}_{20} \pm \frac{10}{77} \sqrt{6} \mathcal{W}_{40} \mp \frac{5}{11} \sqrt{\frac{2}{39}} \mathcal{W}_{60}$ \\
\hline & $\frac{7}{2}$ & 1 & $\frac{7}{2}$ & 1 & $\mathcal{W}_{00}+\frac{\sqrt{5}}{7} \mathcal{W}_{20}-\frac{9}{77} \mathcal{W}_{40}-\frac{15}{11 \sqrt{13}} \mathcal{W}_{60}$ \\
\hline & $\frac{7}{2}$ & 1 & $\frac{7}{2}$ & 2 & $\frac{3}{11} \sqrt{\frac{30}{7}} \mathcal{W}_{44}+\frac{5}{11} \sqrt{\frac{14}{39}} \mathcal{W}_{64}$ \\
\hline & $\frac{7}{2}$ & 2 & $\frac{7}{2}$ & 2 & $\mathcal{W}_{00}-\frac{\sqrt{5}}{21} \mathcal{W}_{20}-\frac{39}{77} \mathcal{W}_{40}+\frac{25}{33 \sqrt{13}} \boldsymbol{W}_{60}$ \\
\hline
\end{tabular}


the $G_{1}^{ \pm}$sector gives the only access to spin- $1 / 2$ phaseshifts $\delta_{\frac{1}{2} 0}$ (for $S_{11}$ ) and $\delta_{\frac{1}{2} 1}$ (for $P_{11}$ ), assuming a spin- 0 meson. However, they mix with $J=3 / 2,5 / 2,7 / 2$ with two-fold multiplicity in $J=7 / 2$. The full mixing with cutoff at $J=7 / 2$ entails a $5 \times 5$ matrix in $\mathcal{M}_{J n, J^{\prime} n^{\prime}}$. The mixing of $J=1 / 2$ with $J=3 / 2$ and $J=5 / 2$ involves $\mathcal{M}_{20}$; while $J=1 / 2$ and $J=7 / 2$ mixing involves $\mathcal{M}_{40}$ and $\mathcal{M}_{44}$. If we assume coupling to $J=7 / 2$ and higher can be ignored, then $\delta_{\frac{1}{2}}$ can be determined via the relation

$$
\left|\begin{array}{ccc}
\mathcal{M}_{\frac{1}{2} \frac{1}{2}}-\cot \delta_{\frac{1}{2}} & \mathcal{M}_{\frac{1}{2} \frac{3}{2}} & \mathcal{M}_{\frac{1}{2} \frac{5}{2}} \\
\mathcal{M}_{\frac{3}{2} \frac{1}{2}} & \mathcal{M}_{\frac{3}{2} \frac{3}{2}}-\cot \delta_{\frac{3}{2}} & \mathcal{M}_{\frac{3}{2} \frac{5}{2}} \\
\mathcal{M}_{\frac{5}{2} \frac{1}{2}} & \mathcal{M}_{\frac{5}{2} \frac{3}{2}} & \mathcal{M}_{\frac{5}{2} \frac{5}{2}}-\cot \delta_{\frac{5}{2}}
\end{array}\right|=0
$$

where the multiplicity and parity labels are suppressed. The determinant in Eq. 9 involves only the product of the three off-diagonal elements and their squares. Two of them $\left(\mathcal{M}_{\frac{1}{2} \frac{3}{2}}\right.$ and $\left.\mathcal{M}_{\frac{3}{2} \frac{5}{2}}\right)$ differ by a sign for even/odd parity, and one the same sign $\left(\mathcal{M}_{\frac{1}{2}}\right)$. This means that Eq. 9 is independent of parity; or $\delta_{\frac{1}{2} 0}$ and $\delta_{\frac{1}{2} 1}$ obey the same phaseshift formula, so do $\delta_{\frac{3}{2} 1}$ and $\delta_{\frac{3}{2} 2}$, and $\delta_{\frac{5}{2} 2}$ and $\delta_{\frac{5}{2} 3}$. So we can suppress the partial-wave $l$ label in $\delta_{J l}$. In fact, the same conclusion extends to the entire $G_{1}^{ \pm}$ sector. If mixing with only $J=3 / 2$ is considered, we have

$$
\boldsymbol{G}_{1}^{ \pm} \text {sector : } \cot \delta_{\frac{1}{2}}=\mathcal{W}_{00}+\frac{\frac{2}{5} \mathcal{W}_{20}^{2}}{\cot \delta_{\frac{3}{2}}-\left(\mathcal{W}_{00}+\frac{1}{\sqrt{5}} \mathcal{W}_{20}\right)}
$$

The determination of spin-1/2 resonances requires $\mathcal{W}_{00}$ and $\mathcal{W}_{20}$ and $\delta_{\frac{3}{2}}$. Only when coupling with $J=3 / 2$ can be ignored can one obtain the simplest formula for the Roper $(P 11)$ and $S_{11}$ phaseshifts $\cot \delta_{\frac{1}{2}}=W_{00}$. On the other hand, if $\delta_{\frac{1}{2}}$ has been independently determined, Eq.10 can be used to access $\delta_{\frac{3}{2}}$.

In the $G_{2}^{ \pm}$sector, the leading contribution is $J=3 / 2$, followed by $J=5 / 2$ and $J=7 / 2$ which both have two-fold multiplicity. The full mixing up to $J=7 / 2$ also entails a $5 \times 5$ matrix in $\mathcal{M}_{J n, J^{\prime} n^{\prime}}$. If we ignore mixing with $J=7 / 2$, the phaseshift relation is given by the $3 \times 3$ matrix equation,

$$
\left|\begin{array}{ccc}
\mathcal{M}_{\frac{3}{2} 1, \frac{3}{2} 1}-\cot \delta_{\frac{3}{2}} & \mathcal{M}_{\frac{3}{2} 1, \frac{5}{2} 1} & \mathcal{M}_{\frac{3}{2} 1, \frac{5}{2} 2} \\
\mathcal{M}_{\frac{5}{2} 2, \frac{3}{2} 1} & \mathcal{M}_{\frac{5}{2} 1, \frac{5}{2} 1}-\cot \delta_{\frac{5}{2}} & 0 \\
\mathcal{M}_{\frac{5}{2} 2, \frac{3}{2} 1} & 0 & \mathcal{M}_{\frac{5}{2} 2, \frac{5}{2} 2}-\cot \delta_{\frac{5}{2}}
\end{array}\right|=0,
$$

which has no coupling between the two multiplicities of $J=5 / 2$. The solution is

$$
\cot \delta_{\frac{3}{2}}=\mathcal{M}_{\frac{3}{2} 1, \frac{3}{2} 1}+\frac{\mathcal{M}_{\frac{3}{2} 1, \frac{5}{2} 1} \mathcal{M}_{\frac{3}{2} 1, \frac{5}{2} 2}}{\cot \delta_{\frac{5}{2}}-\mathcal{M}_{\frac{5}{2} 1, \frac{5}{2} 1}}+\frac{\mathcal{M}_{\frac{5}{2} 2, \frac{5}{2} 2}^{2}}{\cot \delta_{\frac{5}{2}}-\mathcal{M}_{\frac{5}{2} 2, \frac{5}{2} 2}} .
$$

If $J=5 / 2$ can be ignored, one gets the simple phaseshift formula

$$
\boldsymbol{G}_{2}^{ \pm} \text {sector: } \cot \delta_{\frac{3}{2}}=\mathcal{W}_{00}-\frac{1}{\sqrt{5}} \mathcal{W}_{20} .
$$

This gives the best access to the $\Delta$ resonance in the elongated box. On the other hand, $\delta_{\frac{5}{2}}$ can be extracted in this sector if $\delta_{\frac{3}{2}}$ has been independently determined.

\section{Moving states in the elongated box}

So far we have considered two-body states that are at rest; the two particles have back-to-back nonzero momentum, but the total momentum $\boldsymbol{P}=0$ in the lab frame. Now we consider giving the system a 
boost. In the center-of-mass frame (CM) the cubic box becomes a parallelepiped, in which the side parallel to the directions of the boost is contracted by the Lorentz boost factor $\gamma$, whereas the size in the perpendicular direction is unchanged. The advantage of boosting is that it can lower the center-ofmass energy, thus allowing wider access to the resonance region. Table. 4 gives the matrix elements for half-integer $J$ for boosting in the z-direction. The zeta functions now read

$$
\mathcal{W}_{l m}\left(1, q^{2}, \gamma, \eta\right)=\frac{\mathcal{Z}_{l m}^{d}\left(1, q^{2}, \gamma, \eta\right)}{\pi^{\frac{3}{2}} \eta \gamma q^{l+1}}, \mathcal{Z}_{l m}^{d}\left(s, q^{2}, \gamma, \eta\right)=\sum_{\widetilde{\boldsymbol{n}} \in P_{d}(\gamma, \eta)} \frac{\mathcal{Y}_{l m}(\widetilde{\boldsymbol{n}})}{\left(\widetilde{\boldsymbol{n}}^{2}-q^{2}\right)^{s}},
$$

where the summation grid changes to

$$
P_{\boldsymbol{d}}(\gamma, \eta)=\left\{\widetilde{\boldsymbol{n}} \in \mathbb{R}^{3} \mid \widetilde{\boldsymbol{n}}=\hat{\gamma}^{-1} \hat{\eta}^{-1}\left(\boldsymbol{m}+\frac{1}{2} A \boldsymbol{d}\right), \boldsymbol{m} \in \mathbb{Z}^{3}\right\},
$$

with the projector $\hat{\eta}^{-1}$ acting on a vector $\boldsymbol{m}$ to mean $\hat{\eta}^{-1} \boldsymbol{m}=\left(m_{x}, m_{y}, m_{z} / \eta\right)$. Since the boost and elongation are in the same $z$-direction, the factors always appear as a product $\gamma \eta$ in the zeta function, facilitating its evaluation.

Due to lack of parity in boosted states, there is mixing between odd and even $J$ and the entire sector for each irrep becomes coupled. This means that the phaseshift formulas are generally more complicated for moving states than for the ones at rest. In the $G_{2}$ sector, $\delta_{\frac{3}{2} 1}$ and $\delta_{\frac{3}{2} 2}$ become similarly coupled,

$$
\left|\begin{array}{cc}
\mathcal{W}_{00}-\frac{1}{\sqrt{5}} \mathcal{W}_{20}-\cot \delta_{\frac{3}{2} 1} & -i \frac{\sqrt{3}}{5} \mathcal{W}_{10}+i \frac{3}{5 \sqrt{7}} \mathcal{W}_{30} \\
i \frac{\sqrt{3}}{5} \mathcal{W}_{10}-i \frac{3}{5 \sqrt{7}} \mathcal{W}_{30} & \mathcal{W}_{00}-\frac{1}{\sqrt{5}} \mathcal{W}_{20}-\cot \delta_{\frac{3}{2} 2}
\end{array}\right|=0
$$

if we ignore mixing with higher $J$. So the determination of $\delta_{\frac{3}{2} 1}\left(\Delta\right.$ or $P_{33}$ resonance ) requires four zeta functions ( $\mathcal{W}_{l 0}$ with $\left.l=0,1,2,3\right)$ and the knowledge of $\delta_{\frac{3}{2} 2}\left(D_{33}\right.$ resonance). Only when the $\delta_{\frac{3}{2} 2}$ contribution can be ignored do we get the simple formula

$$
\cot \delta_{\frac{3}{2} 1}=W_{00}-\frac{1}{\sqrt{5}} W_{20}
$$

This work is supported in part by the U.S. Department of Energy grant DE-FG02-95ER40907 and the National Science Foundation CAREER grant PHY-1151648. A.A. gratefully acknowledges the hospitality of the Physics Departments at the Universities of Maryland and Kentucky, and the Albert Einstein Center at the University of Bern where part of this work was carried out.

\section{References}

[1] M. Lüscher, Nucl.Phys. B354, 531 (1991)

[2] K. Rummukainen, S.A. Gottlieb, Nucl. Phys. B450, 397 (1995), hep-lat/9503028

[3] Z. Fu, Phys. Rev. D85, 014506 (2012), 1110.0319

[4] L. Leskovec, S. Prelovsek, Phys. Rev. D85, 114507 (2012), 1202 . 2145

[5] M. Gockeler, R. Horsley, M. Lage, U.G. Meissner, P.E.L. Rakow, A. Rusetsky, G. Schierholz, J.M. Zanotti, Phys. Rev. D86, 094513 (2012), 1206.4141

[6] X. Feng, X. Li, C. Liu, Phys.Rev. D70, 014505 (2004), hep-lat/0404001

[7] N. Li, C. Liu, Phys. Rev. D87, 014502 (2013), 1209. 2201

[8] R.A. Briceno, Phys. Rev. D89, 074507 (2014), 1401. 3312

[9] D. Guo, A. Alexandru, R. Molina, M. Döring, Phys. Rev. D94, 034501 (2016), 1605.03993

[10] C. Pelissier, A. Alexandru, Phys.Rev. D87, 014503 (2013), 1211.0092 
Table 4. Non-zero reduced matrix elements for boosting in the cubic box $\left({ }^{2} C_{4 v}\right.$ symmetry group) for half-integral angular momentum up to $J=5 / 2$. There is no multiplicity in the $G_{1}$ sector, but two-fold multiplicity in the $G_{2}$ sector. The horizontal lines separate different combinations of $J J^{\prime}$. The matrix is hermitian in $J l n$ and $J^{\prime} l^{\prime} n^{\prime}$ in each irrep sector.

\begin{tabular}{|c|c|c|c|c|c|c|c|}
\hline$\Gamma$ & $J$ & $l$ & & $J^{\prime}$ & $l^{\prime}$ & & $\mathcal{M}_{J l, J^{\prime} l^{\prime}}^{\Gamma}$ \\
\hline \multirow[t]{16}{*}{$G_{1}$} & $\begin{array}{l}\frac{1}{2} \\
\frac{1}{2}\end{array}$ & $\begin{array}{l}0 \\
0\end{array}$ & & $\begin{array}{l}\frac{1}{2} \\
\frac{1}{2}\end{array}$ & $\begin{array}{l}0 \\
1 \\
\end{array}$ & & $\begin{array}{c}\mathcal{W}_{00} \\
-\frac{i}{\sqrt{3}} \mathcal{W}_{10}\end{array}$ \\
\hline & $\frac{1}{2}$ & 0 & & $\frac{3}{2}$ & 1 & & $i \sqrt{\frac{2}{3}} W_{10}$ \\
\hline & $\frac{1}{2}$ & 0 & & $\frac{3}{2}$ & 2 & & $\sqrt{\frac{2}{5}} \boldsymbol{W}_{20}$ \\
\hline & $\frac{1}{2}$ & 1 & & $\frac{3}{2}$ & 1 & & $-\sqrt{\frac{2}{5}} \boldsymbol{W}_{20}$ \\
\hline & $\frac{1}{2}$ & 1 & & $\frac{3}{2}$ & 2 & & $i \sqrt{\frac{2}{3}} \boldsymbol{W}_{10}$ \\
\hline & $\frac{3}{2}$ & 1 & & $\frac{3}{2}$ & 1 & & $W_{00}+\frac{1}{\sqrt{5}} W_{20}$ \\
\hline & $\frac{3}{2}$ & 1 & & $\frac{3}{2}$ & 2 & & $-i \frac{1}{5 \sqrt{3}} \mathcal{W}_{10}-i \frac{9}{5 \sqrt{7}} \boldsymbol{W}_{30}$ \\
\hline & $\frac{1}{2}$ & 0 & & $\frac{5}{2}$ & 2 & & $\sqrt{\frac{3}{5}} \boldsymbol{W}_{20}$ \\
\hline & $\frac{1}{2}$ & 0 & & $\frac{5}{2}$ & 3 & & $i \sqrt{\frac{3}{7}} \boldsymbol{W}_{30}$ \\
\hline & $\frac{1}{2}$ & 1 & & $\frac{5}{2}$ & 3 & & $-i \sqrt{\frac{3}{7}} W_{30}$ \\
\hline & $\frac{3}{2}$ & 1 & & $\frac{5}{2}$ & 2 & & $i \frac{3 \sqrt{2}}{5} \mathcal{W}_{10}+\frac{2}{5} i \sqrt{\frac{6}{7}} \boldsymbol{W}_{30}$ \\
\hline & $\frac{3}{2}$ & 1 & & $\frac{5}{2}$ & 3 & & $\frac{1}{7} \sqrt{\frac{6}{5}} \boldsymbol{W}_{20}+\frac{2 \sqrt{6}}{7} \boldsymbol{W}_{40}$ \\
\hline & $\frac{3}{2}$ & 2 & & $\frac{5}{2}$ & 2 & & $-\frac{1}{7} \sqrt{\frac{6}{5}} \boldsymbol{W}_{20}-\frac{2 \sqrt{6}}{7} \boldsymbol{W}_{40}$ \\
\hline & $\frac{5}{2}$ & 2 & & $\frac{5}{2}$ & 2 & & $\mathcal{W}_{00}+\frac{8}{7 \sqrt{5}} \mathcal{W}_{20}+\frac{2}{7} \boldsymbol{W}_{40}$ \\
\hline & $\frac{5}{2}$ & 2 & & $\frac{5}{2}$ & 3 & & $-i \frac{\sqrt{3}}{35} \mathcal{W}_{10}-i \frac{8}{15 \sqrt{7}} \mathcal{W}_{30}-i \frac{50}{21 \sqrt{11}} \mathcal{W}_{50}$ \\
\hline & $J$ & $l$ & $n$ & $J^{\prime}$ & $l^{\prime}$ & $n^{\prime}$ & $\mathcal{M}_{J l n, J^{\prime} l^{\prime} n^{\prime}}^{\Gamma}$ \\
\hline \multirow[t]{13}{*}{$G_{2}$} & $\frac{3}{2}$ & 1 & 1 & $\frac{3}{2}$ & 1 & 1 & $\mathcal{W}_{00}-\frac{1}{\sqrt{5}} \boldsymbol{W}_{20}$ \\
\hline & $\frac{3}{2}$ & 1 & 1 & $\frac{3}{2}$ & 2 & 1 & $-i \frac{\sqrt{3}}{5} \mathcal{W}_{10}+i \frac{3}{5 \sqrt{7}} \mathcal{W}_{30}$ \\
\hline & $\frac{3}{2}$ & 1 & 1 & $\frac{5}{2}$ & 2 & 1 & $i \frac{2 \sqrt{3}}{5} \mathcal{W}_{10}-i \frac{6}{5 \sqrt{7}} \mathcal{W}_{30}$ \\
\hline & $\frac{3}{2}$ & 1 & 1 & $\frac{5}{2}$ & 3 & 1 & $\frac{6}{7 \sqrt{5}} \boldsymbol{W}_{20}-\frac{2}{7} \boldsymbol{W}_{40}$ \\
\hline & $\frac{3}{2}$ & 1 & 1 & $\frac{5}{2}$ & 3 & 2 & $-2 \sqrt{\frac{2}{7}} \mathcal{W}_{44}$ \\
\hline & $\frac{3}{2}$ & & 1 & $\frac{5}{2}$ & 2 & 1 & $-\frac{6}{7 \sqrt{5}} \boldsymbol{W}_{20}+\frac{2}{7} \boldsymbol{W}_{40}$ \\
\hline & $\frac{3}{2}$ & 2 & 1 & $\frac{5}{2}$ & 2 & 2 & $2 \sqrt{\frac{2}{7}} W_{44}$ \\
\hline & $\frac{5}{2}$ & 2 & 1 & $\frac{5}{2}$ & 2 & 1 & $\mathcal{W}_{00}+\frac{2}{7 \sqrt{5}} \boldsymbol{W}_{20}-\frac{3}{7} \boldsymbol{W}_{40}$ \\
\hline & $\frac{5}{2}$ & 2 & 1 & $\frac{5}{2}$ & 2 & 2 & $\sqrt{\frac{2}{7}} W_{44}$ \\
\hline & $\frac{5}{2}$ & 2 & 2 & $\frac{5}{2}$ & 2 & 2 & $\mathcal{W}_{00}-\frac{2 \sqrt{5}}{7} \mathcal{W}_{20}+\frac{1}{7} \mathcal{W}_{40}$ \\
\hline & $\frac{2}{2}$ & & 1 & $\frac{2}{2}$ & 3 & 1 & $-i \frac{3 \sqrt{3}}{35} \mathcal{W}_{10}-i \frac{2 \sqrt{7}}{15} \mathcal{W}_{30}+i \frac{25}{21 \sqrt{11}} \mathcal{W}_{50}$ \\
\hline & $\frac{5}{2}$ & 2 & 1 & $\frac{5}{2}$ & 3 & 2 & $5 i \sqrt{\frac{2}{77}} W_{54}$ \\
\hline & $\frac{5}{2}$ & 2 & 2 & $\frac{5}{2}$ & 3 & 2 & $i \frac{\sqrt{3}}{7} \boldsymbol{W}_{10}-i \frac{2}{3 \sqrt{7}} \boldsymbol{W}_{30}+i \frac{5}{21 \sqrt{11}} \boldsymbol{W}_{50}$ \\
\hline
\end{tabular}

